\title{
The Optimal Hedging Ratio for Contingent Claims Based on Different Risk Aversions
}

\author{
Jianhua Guo \\ School of Economics and Management, Shaoyang University, Shaoyang, China \\ Email: jhguo888@163.com
}

How to cite this paper: Guo, J.H. (2019) The Optimal Hedging Ratio for Contingent Claims Based on Different Risk Aversions. Open Journal of Business and Management, 7, 447-454.

https://doi.org/10.4236/ojbm.2019.72030

Received: January 24, 2019

Accepted: March 4, 2019

Published: March 7, 2019

Copyright $\odot 2019$ by author(s) and Scientific Research Publishing Inc. This work is licensed under the Creative Commons Attribution International License (CC BY 4.0).

http://creativecommons.org/licenses/by/4.0/

\section{Open Access}

\begin{abstract}
Based on utility theory, this paper firstly combined different utility functions with risk aversion coefficient and constructed different kinds of optimizing problems for hedgers to hedge for stochastic-payment-typed contingent claim, and then, by the aid of dynamic programming theory, effective multi-stage hedging strategy is proposed for different risk-averse hedgers. Lastly, the research results that the optimal hedging ratios for three kinds of utility functions are equivalent and do not relate to the risk aversion coefficient.
\end{abstract}

\section{Keywords}

Risk Aversion, Contingent Claim, Hedging, The Optimal Hedging Ratio

\section{Introduction}

As an agreement to execute at the terminal moment, contingent claim is actually a measurable and non-negative random variable. And up to now, more and more enterprises introduce contingent claim to bestir their employee. For example, share option is a kind of contingent claim for a listed company to promote employee's operation effectiveness. For non-listed companies, they usually consent that their chief executives may be entitled to buy shares of the company with the present net asset value per-share, thus, if the net asset value per-share arise in future, the chief executives will be rewarded in perquisites.

As for hedging for contingent claims, in a complete market, one may completely hedge a contingent claim by self-financing method; however, in an incomplete market, as to a contingent claim, one can not completely hedge the potential risks. So, how to minimize the potential risks is what investors must think about, and many hedging methods were put forward; among them, Bouleau and Lamberton [1] firstly put forward mean-variance hedging method, which is to minimize the terminal expected value difference between the contin- 
gent claim and hedging positions, i.e., to minimize, after this, many scholars have researched the hedging problem under mean-variance settings. Ales Cerny [2] and Gugushvili S. [3] studied the mean-variance hedging problem by using the dynamic programming theory and acquired the recursive-formatted optimal hedging strategies under discrete time model; Darrell Duffie et al. [4] and Jean Paul Laurent et al. [5] researched the mean-variance hedging problem under diffusion model and acquired the explicit solution of the optimal hedging strategies. Yang and Xiao [6] studied the risk-minimizing hedging problem by using of the Galtchouk-Kunita-Watanabe decomposing method and testified the existence and the Uniqueness of the optimal hedging strategies. Zhang W. et al. [7] and Ni J. et al. [8] studied the hedging problem about contingent claim produced during merchandise transaction.

As far as I know that most of those presented documents about hedging did not take the risk preference of investors into account, however, different investors have different risk preference and different ability of foreseeing financial risk, thus, for different investors, their hedging strategies must be not compatible with each other. In this paper, first off, we combine different utility functions with risk aversion coefficient and construct different kinds of optimizing problems for hedgers to hedge for stochastic-payment-typed contingent claim, and then, by the aid of dynamic programming theory, effective multi-stage hedging strategy is proposed for different risk-averse hedgers

\section{Models}

\subsection{Utility Function}

First, confirm that you have the correct template for your paper size. This template has been tailored for output on the custom paper size $(21 \mathrm{~cm} * 28.5 \mathrm{~cm})$. Risk aversion or risk preference is scale to measure investors' attitude to financial risk, Arrow (1971) [9] pointed out in his reference about financial risk that there were absolute risk aversion coefficient and relative risk aversion coefficient. Assuming that $\mathrm{WN}$ denotes the value of the terminal wealth while $\mathrm{U}(\mathrm{WN})$ denotes a utility function which is continuous and is two-order differentiable, we call $r_{A}\left(W_{N}\right)=-\frac{U^{\prime \prime}\left(W_{N}\right)}{U^{\prime}\left(W_{N}\right)}$ be an absolute risk-hedging coefficient, and $r_{R}\left(W_{N}\right)=-\frac{W_{N} U^{\prime \prime}\left(W_{N}\right)}{U^{\prime}\left(W_{N}\right)}$ be a relative risk-hedging coefficient. If $r_{A}\left(W_{N}\right)>0$ and $r_{R}\left(W_{N}\right)>0$, investors are of risk aversion, and if $r_{A}\left(W_{N}\right)<0$ and $r_{R}\left(W_{N}\right)<0$, investors are of risk preference, or else, if $r_{A}\left(W_{N}\right)=0$ and $r_{R}\left(W_{N}\right)=0$, investors are risk neutral. Under uncertain economic environment, anyone will try her best to maximize the expected utility of her terminal wealth to avoid financial risk [10] [11].

Here, we consider three common utility functions which relate to financial risk:

1) Quadratic utility function : $U\left(W_{N}\right)=W_{N}-\frac{\lambda}{2} W_{N}^{2}$, where $\lambda$ is called as a 
risk hedging coefficient. For a hedger with this quadratic utility function, she is of risk aversion and her risk aversion enhances while her wealth increases. The expected value of utility of wealth can be denotes as $E\left[U\left(W_{N}\right)\right]=E\left[W_{N}\right]-\frac{1}{2} \lambda \operatorname{Var}\left[W_{N}\right]$.

2) Power utility function: $U\left(W_{N}\right)=\frac{W_{N}^{(1-\lambda)}}{1-\lambda}, 0<\lambda<1$, which is also called as relative risk-hedging utility function. When $W_{N}$ is a random variable, the expected utility value can be denoted as $E\left[U\left(W_{N}\right)\right]=\frac{1}{1-\lambda} E\left(W_{N}^{1-\lambda}\right)$.

3) negative exponential utility function: $U\left(W_{N}\right)=-\exp \left(-\lambda W_{N}\right), \quad \lambda>0$, which is also called as absolute risk-hedging utility function and the expected utility value can be denoted as $E\left[U\left(W_{N}\right)\right]=E\left[-\exp \left(-\lambda W_{N}\right)\right]$.

\subsection{The Hedging Model}

Assuming one people, such as an executive chief of a corporation, acquired a copy of contingent claim that will be executed at the terminal moment $\mathrm{T}$ according to his or her work rate, which is called as a kind of Stochastic-PaymentTyped Contingent Claim, in order to acquire the maximal profit, the executive chief, as a hedger, shall use the underlying asset related to the contingent claim to hedge the potential risk at discrete moments $t \in\{0, \Delta t, 2 \Delta t, \cdots, N \Delta t\}$ with time interval $\Delta t=T / N$ during period $[0, T]$.

Let St be the price of contingent claim at $t=n \Delta t$, and $F_{t}$ be the price of the future contract underling on the claim, for a hedger, she use the future contract to hedge the risk of the contingent claim and $\vartheta_{t}$ denotes the position she holds at moment $t=n \Delta t$, while $W_{t}=S_{t}-\vartheta_{t} F_{t}$ denotes the value of hedging asset portfolio at moment $t=n \Delta t$, which will evolve as expressions (1).

$$
W_{t}=\left\{\begin{array}{lc}
W_{0} & t=1 \\
R W_{t-1}-\left(F_{t}-F_{t-1}\right) \vartheta_{t-1} & 2 \leq t \leq N-1 \\
R W_{N-1}-\left(F_{N}-F_{N-1}\right) \vartheta_{N-1}+S_{N} & t=N
\end{array}\right.
$$

where, $R=\exp (r \Delta t)$ and $\mathrm{r}$ denotes the risk-free rate and.

Under the constraint of self-financing as (1), we can construct the hedging model just as following expressions (2).

$$
\max _{\left\{\vartheta_{t}\right\}_{t=1}^{N-1}} E_{1}\left[U\left(W_{N}\right)\right]
$$

where $E_{1}\left[U\left(W_{N}\right)\right]$ is an expected utility function, denoting the expected utility of the terminal wealth, and $E_{t}[\cdot]=E\left[\cdot \mid F_{t}\right], t=1, \cdots, N$ denote the conditional expectation, and similarly, $\operatorname{Var}_{t}[\cdot]=\operatorname{Var}\left[\cdot \mid F_{t}\right], t=1, \cdots, N$ denote the conditional variance.

\section{The Solutions}

\subsection{The Basic Theory}

According to the Bellman principal [12], the expressions (2) can be rewritten 
as (3) as following, and the optimal hedging ratios at each moment $t=n \Delta t$ may be acquired by using the backward recursion method.

$$
\begin{aligned}
& \max _{\left(\vartheta_{1}, \cdots, \vartheta_{N-1}\right)} E_{1}\left[U\left(W_{N}\right)\right] \\
= & \max _{\left(\vartheta_{1}, \cdots, \vartheta_{N-2}\right)} E_{1}\left\{\max _{\vartheta_{N-1}} E_{N-1}\left[U\left(W_{N}\right)\right]\right\} \\
= & \cdots \\
= & \max _{\vartheta_{1}} E_{1}\left\{\max _{\vartheta_{2}} \cdots E\left\{\max _{\vartheta_{N-2}} E\left\{\max _{\vartheta_{N-1}} E\left[U\left(W_{N}\right)\right]\right\}\right\} \cdots\right\}
\end{aligned}
$$

\subsection{The Optimal Hedging Ratio}

Proposition 1: In non-arbitrage market, the optimal hedging ratio of hedging problem $\max _{\left\{\vartheta_{n}\right\}_{n=1}^{N-1}} E_{1}\left[U\left(W_{N}\right)\right]=E_{1}\left(W_{N}\right)-\frac{\lambda}{2} \operatorname{Var}_{1}\left(W_{N}\right)$ may be expressed as

$$
\vartheta_{n}^{*}=\frac{1}{R^{N-n-1}}, n=1, \cdots, N-1
$$

Proof: according to the dynamic programming principal, we can acquire the expressions (4) as following steps.

Step 1, At the moment $t=(N-1) \Delta t$, according to (1), there is

$$
W_{N}=R W_{N-1}-\left(F_{N-1}-F_{N-1}\right) \vartheta_{N-1}+S_{N}
$$

If substitute (5) into $E_{N-1}\left[U\left(W_{N}\right)\right]=E_{N-1}\left[W_{N}\right]-\frac{1}{2} \lambda V a r_{N-1}\left[W_{N}\right]$, we can get the following optimizing problem (6):

$$
\begin{aligned}
& \max _{\vartheta_{N-1}} E_{N-1}\left[U\left(W_{N}\right)\right] \\
= & \max _{\vartheta_{N-1}} E_{N-1}\left[U\left(R W_{N-1}-\left(F_{N}-F_{N-1}\right) \vartheta_{N-1}+S_{N}\right)\right] \\
= & \max _{\vartheta_{N-1}}\left\{E_{N-1}\left[R W_{N-1}-\left(F_{N}-F_{N-1}\right) \vartheta_{N-1}+S_{N}\right]\right. \\
& \left.-\frac{1}{2} \operatorname{Var}_{N-1}\left[R W_{N-1}-\left(F_{N}-F_{N-1}\right) \vartheta_{N-1}+S_{N}\right]\right\}
\end{aligned}
$$

Only need to make derivative calculation on $\vartheta_{N-1}$ in (6), and let the differential coefficient equal to zero, we can acquire the optimal hedging ratio $\vartheta_{N-1}^{*}$ at the moment $t=(N-1) \Delta t$ as following expressions (7).

$$
\vartheta_{N-1}^{*}=\frac{F_{N-1}-E_{N-1}\left(F_{N}\right)+\lambda \operatorname{Cov}_{N-1}\left(F_{N}, S_{N}\right)}{\lambda \operatorname{Var}_{N-1}\left(F_{N}\right)}
$$

When in non-arbitrage market, there $E_{N-1}\left(F_{N}\right)=F_{N-1}$, and because the basic difference between the spot(contingent claim) and the future equals to zero, i.e., $F_{N}=S_{N}$, the (7) may be expressed as (8).

$$
\vartheta_{N-1}^{*}=\frac{0+\lambda \operatorname{Cov}_{N-1}\left(F_{N}, F_{N}\right)}{\lambda \operatorname{Var}_{N-1}\left(F_{N}\right)}=\frac{1}{R^{0}}
$$

At the moment $t=(N-2) \Delta t$, if substitute the optimal hedging ratio just as 
expressions (8) into $W_{N}=R W_{N-1}-\left(F_{N-1}-F_{N-2}\right) \vartheta_{N-1}+S_{N}$, and according to (1), we can get

$$
\begin{aligned}
W_{N} & =R W_{N-1}-\left(F_{N}-F_{N-1}\right) \vartheta_{N-1}^{*}+S_{N} \\
& =R^{2} W_{N-2}-R\left(F_{N-1}-F_{N-2}\right) \vartheta_{N-2}-\left(F_{N}-F_{N-1}\right)+S_{N}
\end{aligned}
$$

Similarly, if put (9) into $E_{N-2}\left[U\left(W_{N}\right)\right]=E_{N-2}\left[W_{N}\right]-\frac{1}{2} \lambda V a r_{N-2}\left[W_{N}\right]$, we can get the optimizing problem (10).

$$
\begin{aligned}
\max _{\vartheta_{N-2}} E_{N-2}\left[U\left(W_{N}\right)\right] \\
=\max _{\vartheta_{N-2}} E_{N-2}\left[U\left(R^{2} W_{N-2}-R\left(F_{N-1}-F_{N-2}\right) \vartheta_{N-2}-\left(F_{N}-F_{N-1}\right)+S_{N}\right)\right] \\
=\max _{\vartheta_{N-2}}\left\{E_{N-2}\left[R^{2} W_{N-2}-R\left(F_{N-1}-F_{N-2}\right) \vartheta_{N-2}-\left(F_{N}-F_{N-1}\right)+S_{N}\right]\right. \\
\left.\quad-\frac{1}{2} \operatorname{Var}_{N-2}\left[R^{2} W_{N-2}-R\left(F_{N-1}-F_{N-2}\right) \vartheta_{N-2}-\left(F_{N}-F_{N-1}\right)+S_{N}\right]\right\}
\end{aligned}
$$

Only need to make derivative calculation on $\vartheta_{N-2}$ in (10), and let the differential coefficient equal to zero, we can acquire the optimal hedging ratio $\vartheta_{N-2}^{*}$ at the moment $t=(N-2) \Delta t$ as following expressions (11).

$$
\begin{aligned}
\vartheta_{N-2}^{*} & =\frac{F_{N-2}-E_{N-2}\left(F_{N-1}\right)-\lambda \operatorname{Cov}_{N-2}\left(F_{N-1}, F_{N}-F_{N-1}\right)+\lambda \operatorname{Cov}_{N-1}\left(F_{N-1}, S_{N}\right)}{\lambda \operatorname{Var}_{N-1}\left(F_{N}\right)} \\
& =\frac{1}{R}
\end{aligned}
$$

Step 2, assume at any moment $t=(n+1) \Delta t$, the optimal hedging ratio can be expressed as $\vartheta_{n+1}^{*}=\frac{1}{R^{N-n-2}}$.

Step 3, we only need to prove that there is $\vartheta_{n}^{*}=\frac{1}{R^{N-n-1}}$ at moment $t=n \Delta t$.

In fact, according to (1), we can get the recursion expressions as following (12).

$$
W_{N}=R^{N-n} W_{n}-\sum_{i=1}^{N-n} R^{N-n-i}\left(F_{n+i}-F_{n+i-1}\right) \vartheta_{n+i-1}+S_{N}
$$

Now, only need to put all $\vartheta_{i}^{*}=\frac{1}{R^{N-i-1}}(i=N-1, \cdots, n+1)$ into (12), there is the optimizing problem (13), and in non-arbitrage market, we can solve (13) and acquire the optimal hedging ratio at moment $t=n \Delta t$ as in (14).

$$
\begin{aligned}
& \max _{\vartheta_{n}} E_{n}\left[U\left(W_{N}\right)\right]=E_{n}\left[W_{N}\right]-\frac{1}{2} \lambda \operatorname{Var}_{n}\left[W_{N}\right] \\
& =E_{n}\left[R^{N-n} W_{n}-R^{N-n-1}\left(F_{n+1}-F_{n}\right) \vartheta_{n}-\sum_{i=2}^{N-n}\left(F_{n+i}-F_{n+i-1}\right)+S_{N}\right] \\
& -\frac{1}{2} \lambda \operatorname{Var}_{n}\left[R^{N-n} W_{n}-R^{N-n-1}\left(F_{n+1}-F_{n}\right) \vartheta_{n}-\sum_{i=2}^{N-n}\left(F_{n+i}-F_{n+i-1}\right)+S_{N}\right]
\end{aligned}
$$




$$
\begin{aligned}
\vartheta_{n}^{*} & =\frac{F_{n}-E_{n}\left(F_{n+1}\right)-\lambda \operatorname{Cov}_{n}\left[F_{n+1}, \sum_{i=2}^{N-n}\left(F_{n+i}-F_{n+i-1}\right)\right]+\lambda \operatorname{Cov}_{n}\left[F_{n+1}, F_{N}\right]}{\lambda R^{N-n-1} \operatorname{Var}_{n}\left(F_{n+1}\right)} \\
& =\frac{0-\lambda \operatorname{Cov}_{n}\left[F_{n+1}, \sum_{i=2}^{N-n}\left(F_{n+i}-F_{n+i-1}\right)-F_{N}\right]}{\lambda R^{N-n-1} \operatorname{Var}_{n}\left(F_{n+1}\right)} \\
& =\frac{0-\lambda \operatorname{Cov}_{n}\left[F_{n+1}, \sum_{i=2}^{N-n}\left(F_{n+i}-F_{n+i-1}\right)-F_{N}\right]}{\lambda R^{N-n-1} \operatorname{Var}_{n}\left(F_{n+1}\right)} \\
& =\frac{0-\lambda \operatorname{Cov}_{n}\left[F_{n+1},-F_{n+1}\right]}{\lambda R^{N-n-1} \operatorname{Var}_{n}\left(F_{n+1}\right)} \\
= & \frac{1}{R^{N-n-1}}
\end{aligned}
$$

Proposition 2: In non-arbitrage market, the optimal hedging ratio for $\max _{\left\{\left\{_{n}\right\}\right\}_{n=1}^{N-1}} E_{1}\left[U\left(W_{N}\right)\right]=\frac{1}{1-\lambda} E_{1}\left(W_{N}^{1-\lambda}\right)$ can be expressed as in (15).

$$
\vartheta_{n}^{*}=\frac{1}{R^{N-n-1}}, n=1, \cdots, N-1
$$

Proof, Firstly, at moment $t=(N-1) \Delta t$, denoting $\lambda^{\prime}=1-\lambda$, then, there is

$$
\max _{\vartheta_{N-1}} E_{N-1}\left[U\left(W_{N}\right)\right]=\frac{1}{\lambda^{\prime}} E_{N-1}\left(W_{N}^{\lambda^{\prime}}\right), \quad 0<\lambda^{\prime}<1
$$

If substituting $W_{N}=R W_{N-1}-\left(F_{N}-F_{N-1}\right) \vartheta_{N-1}+S_{N}$ into (16), and make derivative calculation on $\vartheta_{N-1}$ in (16), and let the differential coefficient equal to zero, there is

$$
\begin{aligned}
& F_{N-1} E_{N-1}\left[\left(R W_{N-1}-\left(F_{N}-F_{N-1}\right) \vartheta_{N-1}+S_{N}\right)^{\gamma^{\prime}-1}\right] \\
& =E_{N-1}\left[F_{N}\left(R W_{N-1}-\left(F_{N}-F_{N-1}\right) \vartheta_{N-1}+S_{N}\right)^{\gamma^{\prime}-1}\right]
\end{aligned}
$$

Assuming the market is complete, i.e., $E_{N-1}\left(F_{N}\right)=F_{N-1}$ and the basic difference between the spot(contingent claim) and the future equals to zero, we can acquire the optimal hedging ratio $\vartheta_{N-1}^{*}$ at the moment $t=(N-1) \Delta t$ as following expressions (18).

$$
\vartheta_{N-1}^{*}=1=\frac{1}{R^{0}}
$$

Then, assume at any moment $t=(n+1) \Delta t$, the optimal hedging ratio can be expressed as $\vartheta_{n+1}^{*}=\frac{1}{R^{N-n-2}}$. now, only need to proof $\vartheta_{n}^{*}=\frac{1}{R^{N-n-1}}$.

In fact, according to (1), we can get the recursion expressions as following (19).

$$
W_{N}=R^{N-n} W_{n}-\sum_{i=1}^{N-n} R^{N-n-i}\left(F_{n+i}-F_{n+i-1}\right) \vartheta_{n+i-1}+S_{N}
$$


Only substitute all $\vartheta_{i}^{*}=\frac{1}{R^{N-i-1}}(i=N-1, \cdots, n+1)$ into (19), there is

$$
W_{N}=R^{N-n} W_{n}-R^{N-n-1}\left(F_{n+1}-F_{n}\right) \vartheta_{n}-\sum_{i=2}^{N-n}\left(F_{n+i}-F_{n+i-1}\right)+S_{N}
$$

Just as acquiring $\vartheta_{N-1}^{*}$, we can solve problem (21),

$$
\begin{aligned}
& \max _{\vartheta_{n}} E_{n}\left[U\left(W_{N}\right)\right]=\frac{1}{\lambda^{\prime}} E_{n}\left[W_{N}^{\lambda^{\prime}}\right] \\
& =\frac{1}{\lambda^{\prime}} E_{n}\left[\left(R^{N-n} W_{n}-R^{N-n-1}\left(F_{n+1}-F_{n}\right) \vartheta_{n}-\sum_{i=2}^{N-n}\left(F_{n+i}-F_{n+i-1}\right)+S_{N}\right)^{\lambda^{\prime}}\right]
\end{aligned}
$$

There is $\vartheta_{n}^{*}=\frac{1}{R^{N-n-1}}$.

Proposition 3: In non-arbitrage market, the optimal hedging ratio for $E\left[U\left(W_{N}\right)\right]=E\left[-\exp \left(-\lambda W_{N}\right)\right]$ can be expressed as in (22).

$$
\vartheta_{n}^{*}=\frac{1}{R^{N-n-1}}, n=1, \cdots, N-1
$$

The prove is similar to proposition 1 .

\section{Conclusion}

In this paper, based on utility theory, we researched the hedging problem for stochastic-payment-typed contingent claim. Firstly, we combined different utility functions with risk aversion coefficient and constructed different kinds of optimizing problems for hedgers to hedge for stochastic-payment-typed contingent claim, and then, by the aid of dynamic programming theory, effective multi-stage hedging strategy is proposed for different risk-averse hedgers. Lastly, the research results that the optimal hedging ratios for different kinds of utility functions are equivalent and do not relate to the risk aversion coefficient.

\section{Fund}

This research is supported by the Social Science Planning Fund Program of Hunan Province of China (Project Number: 17YBA354).

\section{Conflicts of Interest}

The author declares no conflicts of interest regarding the publication of this paper.

\section{References}

[1] Bouleau, N. and Lamberton, D. (1989) Residual Risks and Hedging Strategies in Markovian Markets. Stochastic Processes and their Applications, 33, 131-150. https://doi.org/10.1016/0304-4149(89)90071-9

[2] Cerny, A. (2004) Dynamic Programming and Mean-Variance hedging in Discrete Time. Applied Mathematical Finance, 11, 1-25. https://doi.org/10.1080/1350486042000196164

[3] Gugushvili, S. (2003) Dynamic Programming and Mean-Variance Hedging in Dis- 
crete Time. Georgian Mathematical Journal, 10, 237-246.

[4] Duffie, D. and Richardson, H.R. (1991) Mean-Variance Hedging in Continuous Time. The Annals of Probability, 1, 1-15. https://doi.org/10.1214/aoap/1177005978

[5] Laurent, J.P. and Pham, H. (1999) Dynamic Programming and Mean-Variance Hedging. Finance Stochast, 3, 83-110.

[6] Yang, J.Q. and Xiao, Q.X. (2010) Risk-Minimizing Hedging for Contingent Claims with Stochastic Payments. Applied Mathematics a Journal of Chinese Universities, 25, 1-8.

[7] Zhang, W., Zhou, D. and Liu, L. (2013) Contracts for Changing Times: Sourcing with Raw Material Price Volatility and Information Asymmetry. Manufacturing \& Service Operations Management, 16, 133-148. https://doi.org/10.1287/msom.2013.0454

[8] Ni, J., Chu, L.K., Wu, F., et al. (2012) A Multi-Stage Financial Hedging Approach for the Procurement of Manufacturing Materials. European Journal of Operational Research, 221, 424-431. https://doi.org/10.1016/j.ejor.2012.03.031

[9] Arrow, K.J. (1971) The Theory of Risk Aversion. Essays in the Theory of RiskBearing, 90-120.

[10] Li, Y.H., Wei, Y. and Chen, W. (2014) Application of Risk Aversion to Stock Index Futures Hedging. Journal of Industrial Engineering/Engineering Management, 4, 173-179.

[11] Yao, H.X. and Li, Z.F. (2014) Expected Utility Maximization Optimal Portfolio Selection Based on Non-parametric Estimation Framework. Chinese Journal of Management Science, 22, 1-9.

[12] Lewis, F.L. and Syrmos, V.L. (1995) Optimal Control. 2nd Edition, John Wiley \& Sons, New York, 315. 